\title{
Fistula detection in cerebrospinal fluid leakage ${ }^{1}$
}

\author{
MARShALL B. ALLEN, JR., TAHER EL GAMMAL, MENARD IHNEN, \\ AND MORGAN A. COWAN \\ From the Departments of Surgery and Radiology, Eugene Talmadge Memorial Hospital and the \\ Department of Pathology, University Hospital, Medical College of Georgia, Augusta, \\ Georgia 30902, U.S.A.
}

SUMMARY In two cases of cerebrospinal fluid rhinorrhoea in which scinticisternography failed to identify the fistulae, the tracts were demonstrated by positive contrast ventriculography. It is postulated that the fistula communicated with the ventricles but was isolated from the subarachnoid space by adhesions (demonstrated at operation in one case). There was 'high pressure rhinorrhoea' in one case. The rhinorrhoea ceased after insertion of a ventriculoatrial shunt.

Cisternography utilizing high specific activity radioiodinated human serum albumin (RIHSA) ${ }^{2}$ to demonstrate routes of cerebrospinal fluid (CSF) fistulae and specific techniques for performing such examinations have been described in detail by Di Chiro, Reames, and Matthews (1964, 1968), and Ashburn, Harbert, Briner, and Di Chiro (1968). RIHSA has become popular in the U.S.A. for use in cisternography because it is commercially available, and $\mathrm{Di}$ Chiro and colleagues (1968), in addition to providing detailed descriptions of RIHSA cisternography, have emphasized the safety of the procedure.

Radioisotope cisternography can be used to identify CSF fistulae in patients with recurrent bouts of meningitis who otherwise have questionable clinical or radiological evidence of a fistulous tract. It is also helpful to surgeons planning operative procedures on patients with known rhinorrhoea, since the radioisotope usually produces an identifiable trail which betrays the location of dural defects.

We have recently examined two cases in which obvious CSF fistulae with profuse nasal discharge were not demonstrated using RIHSA cisternography. By utilizing the intraventricular injection of positive contrast medium, their fistulous tracts were demonstrated. One was also demonstrated with intraventricular RIHSA.

\footnotetext{
1 Presented in part at the IVth European Congress of Neurological Surgeons, Prague, Czechoslovakia, 28 June 1971.

2 Source: Abbott Laboratories.
}

The first case is also being described elsewhere in connection with her partially empty sella turcica (El Gammal and Allen, 1970).

CASE 1

R.M., ETMH no. 109-005. This was a 26 year ol obese female with a lifelong history of headaches an ataxia and a one year history of intermittent bug profuse rhinorrhoea. She had been hirsute since she was 8 years old but had experienced menarche at the age of 11 years. Menstrual periods were irregular and she gave a history suggesting an unsuccessful pregnancy.

Physical examination revealed an obese, ataxic, mentally dull, coloured female with an almost constant, watery, nasal discharge. There was a loud systolic bruit heard over both mastoid processes. There was lateral and vertical nystagmus as well as disconjugate eye movements, which later took on the typical characteristics of bilateral internuclear ophthalmoplegia. She had marked impairment of cerebellar function.

Routine laboratory values were within normal limits, as were urinary steroid excretions and blood determinations of thyroid function and follicle stimulating hormone. Radiographs of the skull revealed an enlarged sella turcica and a calcium deposit in the posterior fossa. A brain scan revealed an abnormal concentration of activity in the region of the posterior fossa. Arteriograms revealed a large arteriovenous malformation in the posterior fossa with draining veins extending well above the tentorium (Fig. 1). A radioisotope dynamic flow study, 664 
FIG. 1. Case 1. Late lateral view of an arteriovenous malformation of the posterior fossa demonstrated by left retrograde brachial arteriography. In addition to the primary vascular lesion, note the large draining veins extending into the region of the sella and the parietal and occipital lobes.

utilizing gamma camera scintillation, showed massive blood flow in the posterior fossa consistent with an arteriovenous malformation.

The patient underwent two attempts at scinticisternography via lumbar injection. Recordings were made at three hours, 24 hours, 48 hours, and 72 hours. When these failed to demonstrate a fistula, RIHSA was injected into the right lateral ventricle and a leak into the left frontal sinus was visualized (Fig. 2).

Bifrontal trephines were performed and the patient underwent pneumoencephalography and air and Pantopaque ventriculography which demonstrated the defects produced by draining veins in the region of the third ventricle and an incomplete block of the fourth ventricle. A fistulous tract extending from the left frontal horn to the orbital extension of the left frontal sinus was demonstrated (Fig. 3).

After these examinations, the patient had a ventriculoatrial shunt with arrest of the rhinorrhoea. It recurred two months after discharge when the ventriculoatrial shunt became non-functional but again responded to shunt revision.

COMMENT This case falls into the group classified as high pressure rhinorrhoea by Ommaya, Di Chiro, Baldwin, and Pennybacker (1968). We believe, but have not proved, that the patient has adhesions surrounding the dural defect. Adhesions, so placed, would isolate the fistula from the subarachnoid space. Since the fourth ventricle is partially obstructed to outflow of Pantopaque, and since it probably does not permit flow of fluid from the subarachnoid space into the ventricular system, there is no route by which the radioisotope injected into the subarachnoid space can enter the fistula if the fistulous tract is surrounded by adhesions. Hence, the fistulous tract was demonstrated only when the radioisotope was injected intraventricularly.

CASE 2

H.H., ETMH no. $112-715$, was a 46 year old
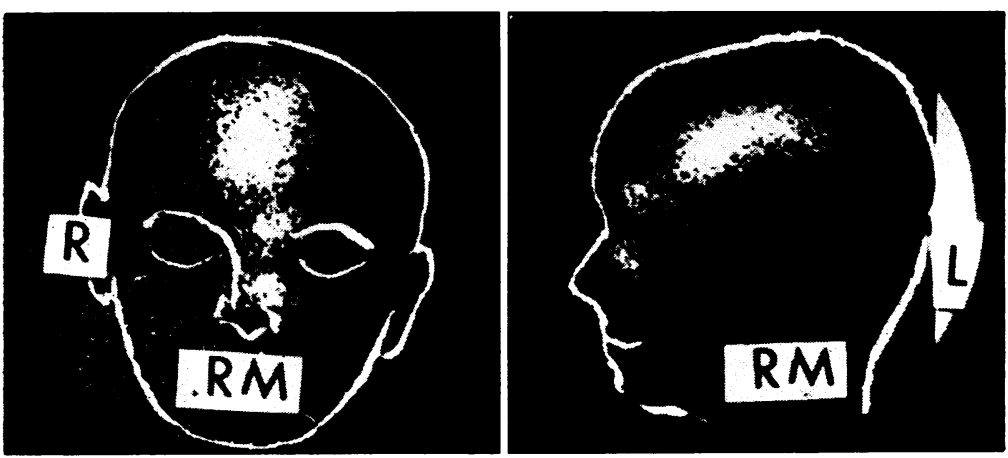

(a) (b)
FIG. 2a and b. Case 1 . RIHSA ventriculogram. Frontal and left lateral recordings were made $5 \frac{1}{2}$ hours after the intraventricular injection of $75 \mu \mathrm{c}$ RIHSA. Note the concentration of isotope in the region of the left frontal sinus. 


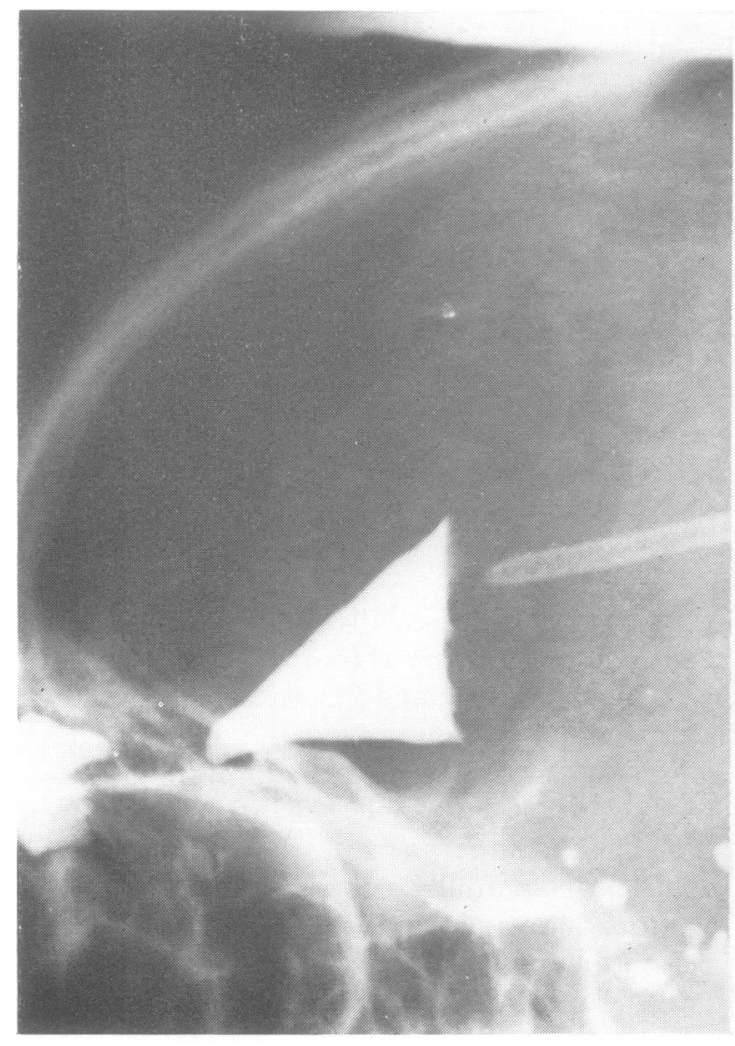

(a)
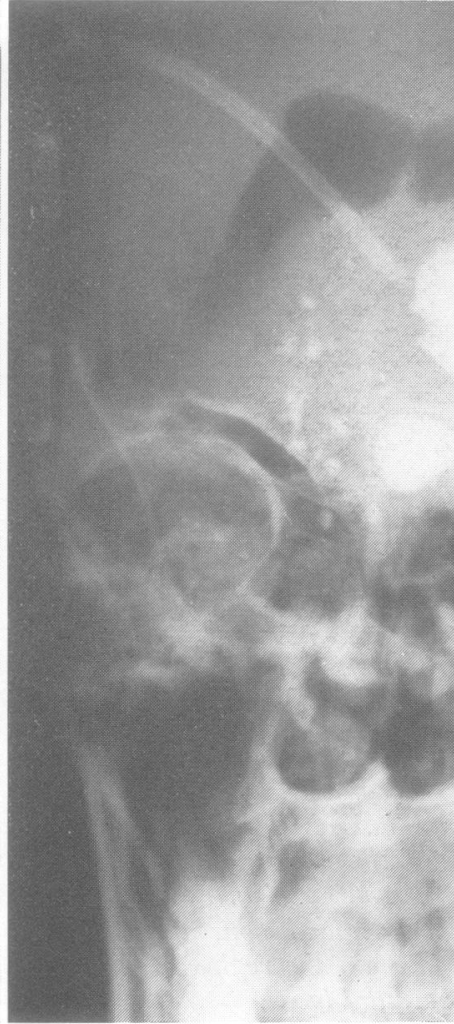

(b)

FIG. 3a and b. Case 1. Ventriculogram after injection of Pantopaque into the left lateral ventricle, demoke strating passage of contrast medium into the left frontal sinus.

labourer who was admitted to the Neurology Service because of headaches and a profuse rhinorrhoea which had persisted since the patient had been struck on the head by a falling tree one year previously. He had been rendered unconscious for about 30 minutes and he had multiple lacerations about the head. Subsequently, he had daily headaches and frequent episodes of a profuse watery, nasal discharge. He had several generalized seizures before admission to our hospital and his wife reported that he had been forgetful and retiring since his injury.

The patient was alert but disoriented and he demonstrated a poor attention span and a profuse rhinorrhoea.

Except for a mildly cellular cerebrospinal fluid (up to 25 cells per c.mm), his laboratory studies were unremarkable. Plain radiographs of the skull revealed a fracture extending into the right frontal sinus, air in the ventricles, and porencephalic cysts. More air was injected into the subarachnoid and ventricular spaces, the better to delineate these $\mathbb{\mathbb { Q }}$ structures. These studies revealed a left to right shift $\overrightarrow{\vec{P}}$ of the midline structures and confirmed the presence of the large porencephalic cysts seen on the plain films.

RIHSA was injected via the lumbar subarachnoid route but the procedure failed to delineate the fistula, although the patient had profuse rhinorrhoea at the time of the examination. Frontal and right lateral scintiphotos were made at three hours and at 25 hours after injection. The patient subsequently had Pantopaque injected into his right lateral ventricle. It passed into the large porencephalic cyst on the right and a fistula extending into the right frontal sinus was demonstrated (Fig. 4). The patient underwent a craniotomy at which time the fistulous tract $\tilde{N}$ was found to be surrounded by adhesions. He has 
subsequently remained free of rhinorrhoea but continued to be disoriented.

COMMENT In this case we likewise failed to demonstrate a fistulous tract by scinticisternography, but we were able to demonstrate the communication by Pantopaque ventriculography. Probably the fistula would have been clearly demonstrated had we obtained an adequate radioisotope ventriculogram.

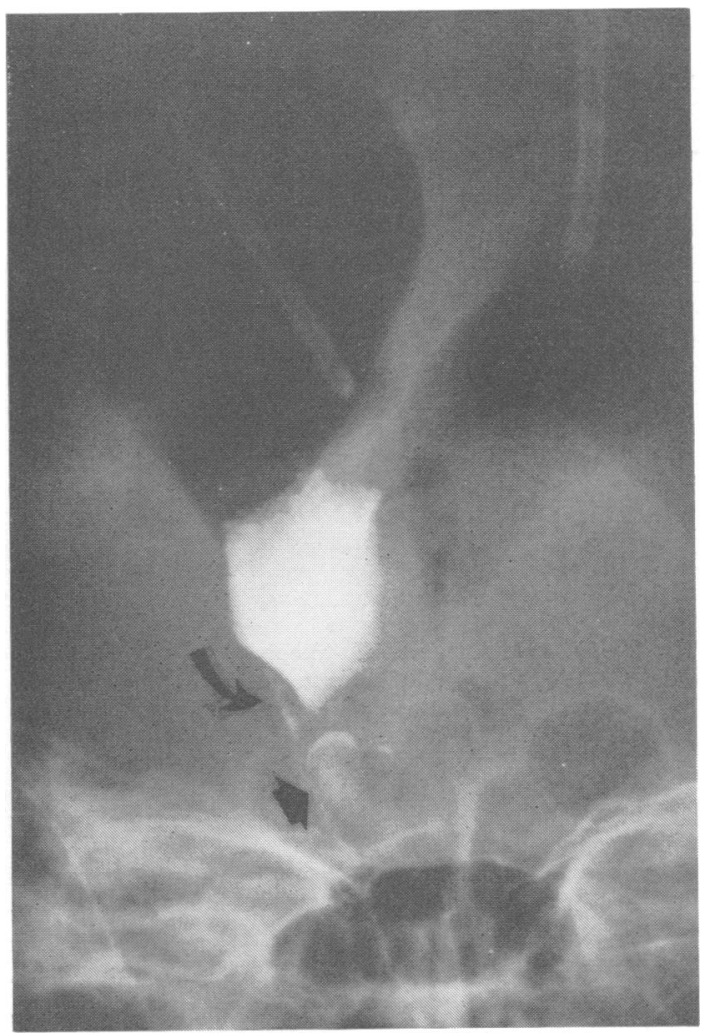

FIG. 4. Case 2. Frontal radiograph made after the injection of Pantopaque into a right lateral ventricle. Note the trail of Pantopaque (curved arrow) from the porencephalic cyst into the frontal sinus (short arrow).

\section{DISCUSSION}

Radiographic identification of CSF fistulae as well as radioisotope cisternography as described by Di Chiro et al. (1964), Ashburn et al. (1968),
Di Chiro, Ommaya, Ashburn, and Briner (1968) can be most useful to the operating neurosurgeon. Positive identification of fistulae before surgery will almost eliminate negative explorations for such lesions and may greatly reduce the amount of trauma which might be required of a surgical procedure to search for the site of fistulous tracts.

In these two cases with profuse rhinorrhoea, we failed to demonstrate the fistulous tract by the usual subarachnoid injection of the radioisotope. Subsequent study in both cases demonstrated a direct communication from the ventricle in one case and communication from the ventricle through a large porencephalic cyst in the other. Adhesions surrounding the fistula were seen at surgery in the second case and were suggested by radiographic examination in the first. Adhesions isolating the fistulous tract from the ventricle to the paranasal sinuses will not permit identification of the fistula by the radioisotopes when injected in the subarachnoid space. Radioisotopes introduced in the subarachnoid space may pass into the ventricular system in cases of extraventricular hydrocephalus (Bannister, Gilford, and Kocen, 1967; Tator, Fleming, Shepherd, and Turner, 1967, 1968; Glasauer, Alber, Leslie, and Nicol, 1968; Glasauer, 1968; Lin, Goodkin, Tong, Epstein, and Vinciguerra, 1968). It is theoretically possible for radioisotopes injected via the subarachnoid route to enter fistulae by passing through the ventricular system, but we do not know how often this occurs when there is normal CSF absorption.

The discreteness with which a positive contrast medium - that is, Pantopaque-outlines defects in ventricular walls and fistulous tracts is apparent in the present cases. Previous reports concerning the use of Pantopaque have indicated its usefulness in demonstrating subarachnoid fistulous tracts, especially from the posterior fossa (Ghouralal, Myers, and Campbell, 1956; Rockett, Wittenborn, Shillito, and Matson, 1964; Pribam, Hass, and Nichioka, 1966; Harrington and Birck, 1967). It has also been reported to demonstrate a fistulous tract after injection into a frontal porencephalic cyst (Jungmann and Peyser, 1963), and it has been injected intranasally to outline a fistula as it flowed intracranially (Teng and Edalatpour, 
1963). We have found one previously reported case where Pantopaque injected intraventricularly revealed a ventriculomastoid fistula (Cantu, Michelsen, and New, 1967).

Our cases have led us to develop a systematic plan for studying patients with CSF rhinorrhoea which we are recommending. The first study would be cisternography carried out in the manner described by Di Chiro et al. (1968) and Ashburn et al. (1968) with frequent periodic scanning of the cranium after isotopes are injected via the lumbar or cisternal route. If this examination is negative, an air study is performed and, in patients who have large ventricles, we recommend insertion of a ventricular cannula and installation of Pantopaque under fluoroscopy with detailed evaluation of the floor of the lateral ventricle on the side of the suspected fistula. If this fails to outline a fistula, we recommend installation of RIHSA through the cannula at the same sitting and proceeding with scanning.

\section{REFERENCES}

Asburn, W. L., Harbert, J. C., Briner, W. H., and Di Chiro, G. (1968). Cerebrospinal fluid rhinorrhoea studied with the gamma scintillation camera. Journal of Nuclear Medicine, 9, 523-529.

Bannister, R., Gilford, E., and Kocen, R. (1967). Isotope encephalography in the diagnosis of dementia due to communicating hydrocephalus. Lancet, 2, 1014-1017.

Cantu, R. C., Michelsen, J. J., and New, P. F. J. (1967). Demonstration of a ventriculo-mastoid fistula (paradoxical cerebrospinal fluid rhinorrhea) by pantopaque ventriculography. Neurochirurgia, 10, 35-44.

Di Chiro, G., Ommaya, A. K., Ashburn, W. L., and Briner, W. H. (1968). Isotope cisternography in the diagnosis and follow-up of cerebrospinal fluid rhinorrhoea. Journal of Neurosurgery, 28, 522-529.

Di Chiro, G., Reames, P. M., and Matthews, W. B., Jr.
(1964). RISA-ventriculography and RISA-cisternography. Neurology (Minneap.), 14, 185-191.

El Gammal, T., and Allen, M. B., Jr. (1970). The intrasellar subarachnoid recess, some clinical and radiological observations. Ninth International Symposium Neuroradiologicum, Gothenburg, Sweden. Acta Radiologica, in press.

Ghouralal, S., Myers, P. W., and Campbell, E. (1956). Persistent cerebrospinal rhinorrhoea originating in a fracture through the petrous bone and cured by muscle graft. Report of a case. Journal of Neurosurgery, 13, 205207.

Glasauer, F. E. (1968). Isotope cisternography and ventriculography. Neurology (Bombay), 16, 143-147.

Glasauer, F. E., Alker, G. J. Jr., Leslie, E. V., and Nicol, C. F. (1968). Isotope cisternography in hydrocephalus with normal pressure. Journal of Neurosurgery, 29, 555-561.

Harrington, J. W., Jr., and Birck, H. G. (1967). Recurrent meningitis due to congenital petrous fistula. A case report. Archives of Otolaryngology, 85, 572-575.

Jungmann, A., and Peyser, E. (1963). Roentgen visualization of cerebrospinal fluid fistula with contrast medium. Radiology, 80, 92-95.

Lin, J. P.-T., Goodkin, R., Tong, E. C. K., Epstein, F. J., and Vinciguerra, E. (1968). Radioiodinated serum albumin (RISA) cisternography in the diagnosis of incisural block and occult hydrocephalus. Radiology, 90, 36-41.

Ommaya, A. K., Di Chiro, G., Baldwin, M., and Pennybacker, J. B. (1968). Non-traumatic cerebrospinal fluid rhinorrhoea. Journal of Neurology, Neurosurgery, and Psychiatry, 31, 214-225.

Pribram, H. F. W., Hass, A. C., and Nishioka, H. (1966). Radiographic localization of a spontaneous cerebrospinat fluid fistula. Case report. Journal of Neurosurgery, 24, $1031-$ 1033.

Rockett, F. X., Wittenborg, M. H., Shillito, J., Jr., and Matson, D. D. (1964). Pantopaque visualization of a congenita dural defect of the internal auditory meatus causing rhinorrhoea. Report of a case. American Journal of Roentgenology, Radium Therapy and Nuclear Medicine, 91 640-646.

Tator, C. H., Fleming, J. F. R., Sheppard, R. H., and Turner V. M. (1967). Studies of cerebrospinal fluid dynamics with intrathecally administered radioiodinated human serum albumin ( ${ }^{131}$ IHSA). Canadian Medical Association Journal, 97, 493-503.

Tator, C. H., Fleming, J. F. R., Sheppard, R. H., and Turner, V. M. (1968). A radioisotopic test for communicating hydrocephalus. Journal of Neurosurgery, 28, 327-340.

Teng, P., and Edalatpour, N. (1963). Cerebrospinal fiuid rhinorrhea with demonstration of cranio-nasal fistula with Pantopaque. Radiology, 81, 802-806 